\title{
Evolution au cours d'une année des quantités de foin ingérées par des béliers castrés
}

\author{
Brigitte MICHALET-DOREAU et F. GATEL \\ avec la collaboration technique de L. L'Hotelier et $\mathrm{H}$. BousqueT \\ I.N.R.A., C.R.Z.V. de Theix, Laboratoire des Aliments \\ F 63122 Ceyrat
}

\begin{abstract}
Résumé
On détermine l'ingestibilité d'un aliment en mesurant la quantité de matière sèche ingérée par le mouton "Standard» dont on suppose la capacité d'ingestion constante. Il semblerait qu'il existe des variations saisonnières de la capacité d'ingestion liées à la photopériode. Pour quantifier ce phénomène dans les conditions d'utilisation du mouton standard, nous avons mesuré pendant un an (de novembre à novembre) les quantités de foin volontairement ingérées par un lot de 6 moutons.

Les quantités ingérées restent stables pendant les trois premiers mois, augmentent (33 p. 100) en mars et avril, puis se stabilisent jusqu'à la fin de l'expérience. Cette ćvolution résulte vraisemblablement d'une part de l'augmentation de la durée d'éclairement et d'autre part de l'adaptation des animaux au foin.
\end{abstract}

\section{Introduction}

L'ingestibilité des aliments est déterminée par la quantité de matière sèche volontairement ingérée par un mouton «standard» (bélier castré de race Texel, âgé de 1 à 4 ans, et pesant de 40 à $90 \mathrm{~kg}$ ) ce qui suppose que la capacité d'ingestion de cet animal soit à peu près constante, ou du moins que ses variations soient connues.

Gordon (1964) a, le premier, mis en évidence chez des brebis ou des béliers castrés une variation saisonnière des quantités ingérées. Par la suite, de nombreux auteurs (Tartelin, 1968 ; Milne et al., 1976 et 1978 ; Kay, 1979 ; Sykes, Coop \& Rushton, 1980 ; Blaxter, Fowler \& Gill, 1982) ont confirmé que les quantités ingérées variaient dans le même sens que la longueur du jour et étaient plus élevées en été qu'en hiver.

Les expériences de El Shahat et al. (1974), Forbes et al. (1979 et 1981) sur des agneaux et de Perers et al. (1981) sur des vaches laitières, dans lesquelles des animaux étaient soumis à deux durées d'éclairement différentes, ont mis en évidence que les quantités ingérées variaient dans le même sens que la photopériode. Pollock (1975) et KAY (1979) ont également montré, avec des animaux soumis à un cycle photopériodique de six mois, le rôle de la photopériode sur la quantité ingérée. 
Il nous a donc paru intéressant de préciser quelle est l'influence de la saison sur la capacité d'ingestion du mouton «standard »utilisé au Laboratoire des Aliments pour mesurer l'ingestibilité des fourrages.

\section{Matériel et méthodes}

Pendant une année (novembre 1980 à novembre 1981) six béliers castrés, âgés de 4 ans et pesant en début d'expérience $70,4 \pm 3,0 \mathrm{~kg}$ ont été placés en cages individuelles, dans un bâtiment fermé, éclairé en lumière naturelle et chauffé l'hiver. La température et l'humidité sont restées comprises entre respectivement 11 et $19{ }^{\circ} \mathrm{C}$, et 50 et 80 p. 100 , et ne présentent pas de variation systématique pendant la période étudiée. Les animaux ont été tondus le 23 avril.

\section{TableaU 1}

Composition chimique et digestibilité (dactyle $-1^{\text {‘r }}$ cycle).

Chemical composition and digestibility of studied forages.

\begin{tabular}{|c|c|c|c|c|}
\hline & $\begin{array}{c}30-11 \\
\text { au } 5-12\end{array}$ & $\begin{array}{l}22-2 \\
\text { au } 27-2\end{array}$ & $\begin{array}{c}13-9 \\
\text { au } 18-9\end{array}$ & $\begin{array}{c}15-11 \\
\text { au } 20-11\end{array}$ \\
\hline $\begin{array}{l}\operatorname{MS}(\%) \\
D M(\%)\end{array}$ & 88,1 & 88,3 & 88,5 & 90,0 \\
\hline $\begin{array}{l}\text { Matière organique (\% MS) } \ldots \ldots \ldots \\
\text { Organic matter }(\% \text { DM })\end{array}$ & 90,8 & 91,1 & 90,6 & 90,1 \\
\hline $\begin{array}{l}\text { Matières azotées ( } \% \text { MS) } \\
\text { Crude protein (\% DM) }\end{array}$ & 10,6 & 10,5 & 11,0 & 10,6 \\
\hline $\begin{array}{l}\text { Ceilulose brute (\% MS) } \ldots \ldots \ldots \ldots \\
\text { Crude fibre (\% DM) }\end{array}$ & 32,8 & 33,6 & 34,2 & 32,3 \\
\hline $\begin{array}{l}\text { Digestibilité de la matière organique } \\
(\%) \ldots \ldots \ldots \ldots \ldots \ldots \ldots \ldots \ldots \ldots \ldots \ldots \ldots \\
\text { Organic matter digestibility (\%) }\end{array}$ & 58,7 & 59,6 & 60,4 & 61,1 \\
\hline
\end{tabular}

Les animaux ont reçu pendant toute l'année (deux distributions par jour à 8 et $16 \mathrm{~h}$ ), un même foin de dactyle récolté au premier cycle au stade fin épiaison, dont les caractéristiques sont données dans le tableau 1. Ce foin a été choisi de manière à couvrir les besoins énergétiques et azotés des animaux. Pour maintenir constant l'état nutritionnel des animaux et en particulier éviter qu'ils ne s'engraissent, nous avons fait alterner, par séquences de deux semaines, les périodes où le foin était distribué à volonté (10 p. 100 de refus) et celles où il était distribué en quantité limitée (40 à 100 p. 100 des quantités ingérées à volonté, selon les animaux et les périodes), de façon à maintenir sur quatre semaines un niveau alimentaire moyen voisin de celui de l'entretien. Lors de chaque période d'alimentation à volonté, les animaux ont été pesés et nous avons mesuré pour chaque animal les quantités de matière sèche ingérées. 


\section{Résultats et discussion}

Pendant la durée de l'expérience, les animaux ont perdu du poids $(5 \mathrm{~kg})$, cette diminution survenant brutalement, dans les premières semaines qui suivent la tonte; par contre, les poids restent relativement stables avant et après la tonte.

Lors des périodes d'alimentation à volonté, nous avons constaté de fortes variations journalières des quantités ingérées (fig. 1); ces variations sont dues pour une part au passage d'une alimentation en quantité limitée à une alimentation à volonté. Faute de pouvoir déterminer exactement à partir de ces résultats la durée de la période d'adaptation pour passer de l'alimentation en quantité limitée à celle à volonté, nous l'avons fixée pour toutes les périodes de mesure, à sept jours, et n'avons considéré que les valeurs de quantité ingérée à partir du huitième jour.

Entre novembre 1980 et novembre 1981, les quantités ingérées moyennes passent de $1145 \pm 167$ à $1617 \pm 233$ g MS par animal et par jour, soit une augmentation de 41 p. 100 : elles restent relativement stables ( $1177 \mathrm{~g}$ MS) jusqu'à la fin du mois de février, augmentent d'environ $184 \pm 32 \mathrm{~g}$ MS par mois entre début mars et début mai, puis se stabilisent (1562 g MS) entre le milieu du mois de mai ct la fin du mois de novembre (fig. 2).

Or ce maximum se situe juste après la tonte. Avec des animaux èn cage, WonZicka-ToMASZWESKa (1963 et 1964) et Minson \& TerNouth (1971) ont constaté, dans certains cas, une augmentation des quantités ingérées qui peut atteindre 30 p. 100 dans les semaines qui suivent la tonte, l'effet de la tonte n'étant plus significatif après cinq ou six semaines. Dans lexpérience présente, la tonte pourrait avoir amplifié momentanément le phénomène d'augmentation des quantités ingérées, dont le maximum «réel » se situerait alors à la fin du mois daoût, ou ne pas avoir eu d'effet, le maximum «réel», se situant alors effectivement à la mi-mai.

Par ailleurs, les variations de quantités ingérées entre animaux qui sont relativement faibles en début d'expérience (coefficient de variation de 3,5 p. 100) augmentent progressivement au cours des dix premières semaines (le coefficient de variatıon restant ensuite compris entre 10 et 17 p. 100 jusqu'à la fin de l'expérience).

Les caractéristiques du foin utilisé n’ont pas varié de façon importante au cours de l'expérience (tabl. 1) et ne peuvent donc expliquer l'évolution des quantités ingérées.

Par contre, la période d'augmentation des quantités ingérées coïncide avec la période d'augmentation de la durée d'éclairement (fig. 2), mais les quantités ingérées restent élevées pendant la phase décroissante de longueur du jour. L'augmentation observée ici (41 p. 100) est légèrement inférieure à celle $(50$ p. 100) rapportée par KAY (1979), peut-être parce que nous avons travaillé sur des animaux castrés, moins sensibles à la durée d'éclairement que les mâles entiers (KAY, 1979). Par contre, elle est supérieure à celles (respectivement 18 à 29 p. 100 et 13 à 30 p. 100) rapportées par Milne et al. (1978), et BlaXter, Fowler \& Gill (1982); l'augmentation des quantités ingérées pourrait être la résultante de l'effet de la photopériode et d'un phénomène d'adaptation des animaux; ceci expliquerait également que les quantités ingérées restent élevées pendant la phase décroissante de longueur du jour, les deux mécanismes ayant des actions contraires. Cette adaptation des animaux pourrait être : 


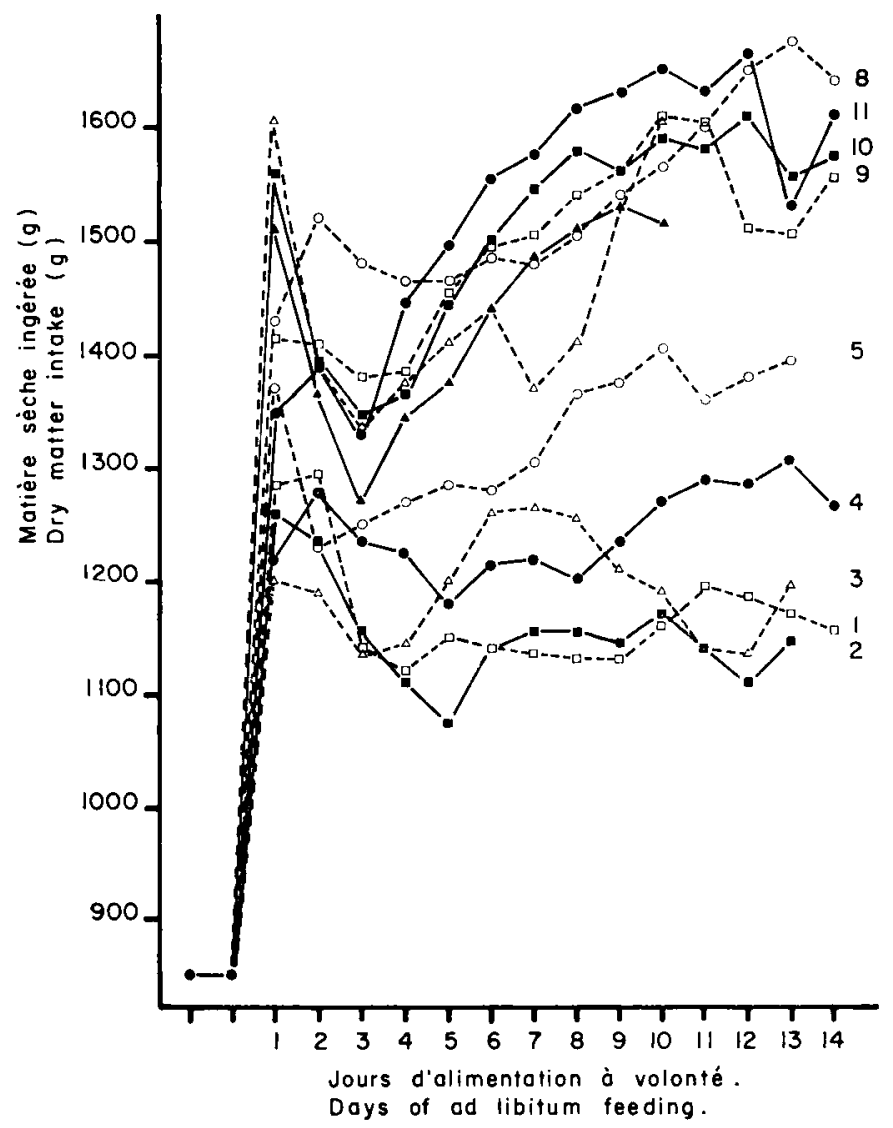

FIG. 1

Evolution journalière des quantités moyennes de matière sèche ingérée au cours de chaque période.

Daily variation of mean voluntary dry matter intake.

1. Période du 20-12 au 2-1. Period from 20-12 to 2-1.

2. Période du 19-1 au 31-1. Period from 19-1 to 31-1.

3. Période du 15-2 au 27-2. Period from 15-2 to 27-2.

4. Période du 14-3 au 27-3. Period from 14-3 to 27-3.

5. Période du 11- : au 23-4. Period from 11-4 to 23-4.

6. Période du 5-6 au 14-6. Period from 5-6 to 14-6.
7. Période du 6-7 au 15-7. Period from 6-7 to 15-7.

8. Période du 8-8 au 21-8. Period from 8-8 to $21-8$.

9. Période du 5-9 au 18-9. Period from 5-9 to $18-9$.

10. Période du 4-10 au 17-10. Period from 4-10 to 17-10.

11. Période du 1-11 au 14-11. Period from 1-11 to 14-11. 


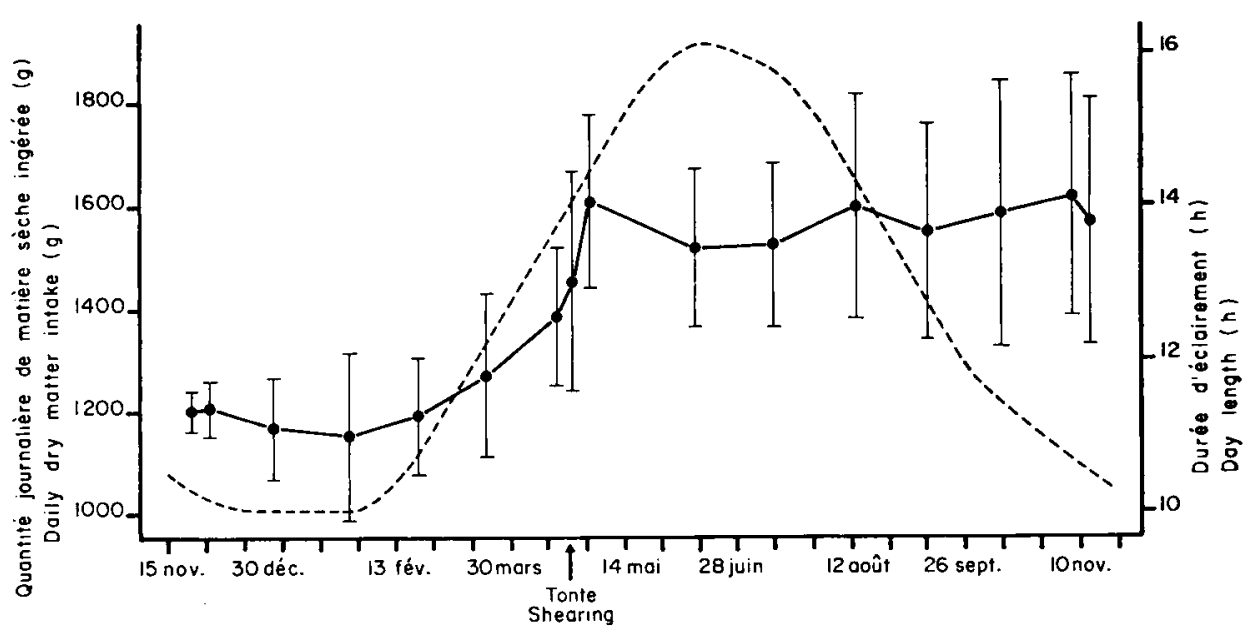

FIG. 2

Evolution des quantités ingérées et de la durée d'éclairement.

Variations of voluntary food intake and day length.

- - Quantité journalière de matière sèche ingérée (g).

Daily dry matter intake $(g)$.

- - - Durée d'éclairement.

Day length.

- une adaptation aux modalités de distribution de fourrage (succession de périodes où l'aliment est distribué à volonté ou en quantité limitée) ;

- ou une adaptation au fourrage reçu; dans les mêmes conditions expérimentales, XANDE (1978) rapporte, avec de la paille, une augmentation importante des quantités ingérées qui doublent en 21 semaines. Dans notre essai on observe d'ailleurs que la digestibilité de la matière organique du foin distribué a eu tendance à augmenter (de 58,7 à 61,1 p. 100) bien que sa composition chimique soit restée constante, alors qu'on aurait pu s'attendre à l'inverse compte tenu de l'augmentation importante des quantités ingérées.

\section{Conclusion}

L'augmentation des quantités ingérées constatées dans cet essai est vraisemblablement la résultante de deux phénomènes : augmentation de la durée d'éclairement et adaptation des animaux au foin ou aux modalités de distribution de ce dernier; mais les conditions expérimentales de notre essai ne nous permettent pas de conclure nettement quant à l'importance respective de ces deux facteurs. 


\section{Summary \\ Annual variations in voluntary food intake of wethers fed cocksfoot hay}

Voluntary food intake is usually determined by measuring the amount of dry matter ingested by a «standard» wether assumed to have a constant ingestion capacity. There is evidence that there are seasonal changes in food intake in relation to daylength. In order to evaluate the effect of daylength on voluntary food intake of «standard wethers 》, food intake was measured for a period of one year (november to november) using six wethers offered cocksfoot hay ad libitum.

Mean daily food intake was steady during the first three months. However, there was a 33 p. 100 increase in food consumption during the course of march and april when daylength was markedly increased. During the final phase of the experiment, food consumption was relatively constant despite a decrease in daylength (fig. 2). The results indicate that the variation in food intake is probably due to both variation in daylength and adaptation of the animal to the food offered.

\section{Références bibliographiques}

Blaxter K.L., Fowler V.R., Gill J.C., 1982. A study of the growth of sheep to maturity. J. agric. Sci. Camb., 98, 405-420.

El Shahat A.A., Jones R., Forbes J.M., Boatz T.G., 1974. The effect of day length on the growth of lambs at two levels of feeding. Proc. Nutr. Soc., 33, 83-84 A.

Forbes J.M., El Shahat A.A., Jones R., Duncan J.G.S., Boatz T.G., 1979. The effect of day lenghth on the growth of lambs. Anim. Prod., 29, 33-42.

Forbes J.M., Brown W.B., Al-Banna A.G.M., Jones R., 1981. The effect of day length on the growth of lambs. Anim. Prod., 32, 23-28.

Gordon J.G., 1964. Effect of time of the year on the roughage intake of housed sheep. Nature, 204, 798-799.

KAY R.N.B., 1979. Seasonal changes of appetite in deer and sheep. ARC Res. Rev., $5,13-15$.

Milne J.A., Mac Rae J.C., Spence A.M., Wilson S., 1976. Intake and digestion of hill land vegetation by the red deer and the sheep. Nature, 263, 763 .

Milne J.A., Mac RaE J.C., Spence A.M., Wilson S., 1978. A comparison of the voluntary intake and digestion of a range of forages at different times of the year by the sheep and the red deer (Cervus elaphus). Br. J. Nutr., 40, 347-356.

Minson D.J., Ternouth J.M., 1971. The expected and observed changes in the intake of three hays by sheep after shearing. Br. J. Nutr., 26, 31-39.

Peters R.R., Chapin L.T., Emery R.S., Tucker H.A., 1981. Milk yield, feed intake, prolactin, growth hormone and glucocorticoid response of cows to supplemented light. J. Dairy Sci., 64, 1671-1678.

Pollock A.M., 1975. Seasonal changes in appetite and sexual condition in red deer stags maintained on a six month photoperiod. J. Physiol., 244, 95-96.

Sykes A.R., CoOP R.L., Rushton B., 1980. Chronic and clinical fascioliasis in sheep : effects on food intake, food utilisation and blood constituents. Res. vet. Sci., 28, 63-70.

Tartelin M.F., 1968. Cyclical variations in food and water intakes in ewes. $\boldsymbol{J}$. Physiol., $195,29-31$.

WoDZICKA-TOMASZEWSKa M., 1963. The effect of shearing on the appetite of sheep. N.Z.J. agric. Res., 6, 440-447.

WodzicKa-TomaszewSKa M., 1964. The effects of shearing on the appetite of two tooth ewes. N.Z.J. agric. Res., 7, 654-662.

XANDE A., 1978. Valeur alimentaire des pailles de céréales chez le mouton. I - Influence de la complémentation azotée et énergétique sur l'ingestion et l'utilisation digestive d'une paille d'orge. Ann. Zootech., 27, 583-599. 